\title{
Modelo para determinar los componentes de la cartera hipotecaria en la Banca Múltiple en el Perú 2001 - 2015
}

\author{
Model to determine the components of the mortgage portfolio in the Multiple Bank in Peru \\ $2001-2015$
}

\author{
Carlos Alberto Guerrero López ${ }^{1}$
}

\begin{abstract}
Resumen
El objetivo de esta investigación fue establecer un modelo que explique la morosidad a través de variables macroeconómicas, tales como la variación del Producto Bruto Interno (PBI) real, la tasa activa en moneda nacional, la tasa activa en moneda extranjera la variación del tipo de cambio real; basado en datos teóricos como empíricos. Siendo la crisis internacional y los problemas externos del precio de los minerales, los que han originado la caída de las exportaciones, motivando que el crecimiento de la economía disminuya; ello llevó a que los agentes económicos que se han financiado por medio de deudas se vean con dificultades para hacer frente a sus compromisos de pago, debido al deterioro de sus ingresos; de esta manera surje el problema del deterioro de la calidad de la cartera morosa; que representa un problema para el sistema bancario y para la economía en general. Siendo la morosidad de la cartera hipotecaria determinada por las variables crecimiento económico, tasas de interés y tipo de cambio. Por ello, conocer las variables que explican la morosidad de las carteras hipotecarias permite anticiparce y minimizar los efectos que se presenten como consecuencia del contexto desfavorable en que se encuentre la economía; así como evaluar las políticas ha ser adoptadas, por las instituciones financieras, para evitar los problemas de liquidez que se presenten a futuro.
\end{abstract}

Palabras Clave: cartera hipotecaria; morosidad; racionamiento del crédito; información imperfecta; inteligencia financiera; tipos de interés; ciclo económico; niveles de endeudamiento; cointegración, insolvencia.

\begin{abstract}
The objective of this research was to establish a model that explains delinquency through macroeconomic variables, such as the variation of the real Gross Domestic Product (GDP), the active rate in national currency, the active rate in foreign currency, the variation in the type of real change; based on theoretical data as empirical. Being the international crisis and the external problems of the prices of the minerals, those that have originated the fall of the exports, motivating that the growth of the economy diminishes; This led to the economic agents that have been financed by means of debts having difficulties in meeting their payment commitments, due to the deterioration of their income; in this way, the problem of the deterioration of the quality of the delinquent portfolio arises; which represents a problem for the banking system and for the economy in general. Being the delinquency of the mortgage portfolio determined by the variables economic growth, interest rates and exchange rate. Therefore, knowing the variables that explain the delinquency of mortgage portfolios allows anticipating and minimizing the effects that arise as a result of the unfavorable context in which the economy finds itself; as well as evaluating the policies to be adopted, by financial institutions, to avoid liquidity problems that arise in the future.
\end{abstract}

Keywords: mortgage portfolio; delinquency; credit rationing; imperfect information; financial intelligence; interest rates; economic cycle; levels of indebtedness.

\section{Introducción}

En los últimos años la morosidad en el Perú ha venido incrementándose; de 1,54\% en enero del 2012 a 2,54\% en diciembre del 2015 (SBS, 2016), Sin considerar la crisis internacional que comenzó a fines de 2007 y alcanzó su punto culminante en el último trimestre del año 2008, cuando el precio de la vivienda dejó de subir y comenzó a bajar rápidamente. El crédito se contrajo considerablemente y los bancos fueron incapaces de utilizar colaterales (las propiedades) como instrumento de recuperación de las deudas (Tanzi, 2010). Los agentes que compraron viviendas dejaron de pagar sus hipotecas, que incidió en el alza de la morosidad respecto a los años anteriores, creando dificultades para los propietarios de instrumentos financieros, a menudo clasificados AAA, que estaban respaldados por la hipoteca. Esto obligó a las instituciones financieras a aumentar su gasto en provisiones para la cartera hipotecaria, y ver el frenético 
deterioro de sus carteras de crédito. En el caso del Perú existe una mejora de la morosidad ya que en el año 1998 era $8,33 \%$ y para el año 2013 fue $2,14 \%$, lo que se debió a la realización de castigos de cartera y liquidaciones de entidades financieras; subiendo a $2,47 \%$ en el 2,014 por la desaceleración de la economía; lo que afectó el pago de los agentes económicos. Esto se puede deber a la desaceleración de la economía mundial, siendo el Perú no ajeno ello, debido a que el menor crecimiento mundial conlleva a menores exportaciones, por lo que la economía crece menos. Esto se puede apreciar al analizar desde el período 2001 al 2012, reduciéndose a $2.14 \%$ habiendo crecido la economía al ritmo de $5 \%$ en promedio durante ese período.

Las formas en que se manifiesta el problema están dadas mediante el deterioro de la calidad de la cartera morosa, esto se debe a que los bancos no mantienen políticas de créditos cautelosas. Por ello, la cartera morosa tiende a aumentar, es decir se deteriora la calidad de la cartera morosa. Es así, que una institución que empieza a sufrir un deterioro de su portafolio de créditos, ve perjudicada su rentabilidad al aumentar la proporción de créditos con intereses no pagados, además de generarse una ruptura en la rotación de los fondos. El problema en la rentabilidad se profundiza al tomar en cuenta que la entidad regulada tiene que incrementar sus provisiones por los créditos impagos, lo que repercute inmediatamente en las utilidades de la empresa. De esta manera, un incremento importante en la morosidad hace que el problema de incumplimiento se traduzca en uno de rentabilidad y liquidez, y finalmente en un problema de solvencia si la institución empieza a generar pérdidas y déficit de provisiones (Guillen, 2001).

Otro estudio manifiesta que un deterioro de la calidad de la cartera conlleva a una reclasificación del cliente a una categoría de mayor riesgo. Esto se debe a que se ve afectado el nivel de patrimonio mínimo requerido por el ente supervisor y así obliga a las entidades a tener una mayor nivel de provisiones, las cuales suponen un porcentaje fijo del total del crédito concedido, lo cual servirá como un fondo para hacer frente a la deuda en caso de impagos (Damodar \& Dawn, 2010). Además se debe tener en consideración los problemas acontecidos en economías desarrolladas denominada crisis subprime (Maubré, 2008).

Debido a la importancia del tema como sostienen (Aguilar et al., 2004) las instituciones financieras tienen como uno de sus objetivos principales mantener y mejorar el nivel de morosidad de sus carteras. Más aún si existen otros investigadores como Chavarín (2015) quien sostiene que el índice de morosidad de los prestatarios se vuelve una variable que impacta de manera negativa y significativa a la rentabilidad obtenida por los bancos.

Conocer las variables que explican la morosidad, permitirá implementar medidas específicas que permitan anticipar y/o minimizar los efectos que se presenten como consecuencia del contexto desfavorable en que nos encontramos; así como evaluar las políticas adoptadas por las instituciones financieras para evitar el problema de liquidez que se presenten, la insolvencia posterior $y$ finalmente el cierre de dicha institución. Estos mismos autores indican que los estudios sobre este tema han sido abordados a partir del análisis de otros problemas relacionados con las entidades financieras, pero no han sido tratadas en la literatura como un problema en sí mismo, es decir analizado de manera exclusiva. Por ello, si investigamos este problema, el estudio se constituiría en una herramienta que contribuiría al entendimiento del problema.

El volumen de créditos hipotecarios de la Banca Múltiple en el Perú ha aumentado tanto en términos absolutos como relativos respecto al total de la cartera; en este sentido este estudio se justifica a fin de buscar una solución al problema de la morosidad crediticia en la banca peruana.

Este estudio busca identificar los factores que determinan la morosidad de los créditos hipotecarios de la banca múltiple peruana, se analizará las variables macroeconómicas en el último quinquenio, tales como: nivel de actividad económica, depreciación del tipo de cambio, tasas de interés activas en moneda nacional y tasas de interés en moneda extranjera que impactan en la morosidad de la cartera. Taambién se estudiará los efectos que generen los deudores hipotecarios el cambio en el ciclo económico, de una fase expansiva hacia una restrictiva, tales como problemas de liquidez mediante el cambio en las tasas de interés activas en moneda nacional y extranjera que afectan la capacidad de los mismos de honrar sus obligaciones. Finalmente como la depreciación de la moneda nacional, mediante la variación del tipo de cambio, afectará a los deudores hipotecarios cuyos créditos están denominados en moneda extranjera mediante un descalce cambiario.

\section{Materiales y métodos}

Es importante realizar unas definiciones como la de:

Los créditos hipotecarios, se define como como un producto financiero que permite a las familias obtener un financiamiento de largo plazo, con el fin de hacerse de un bien inmueble que se constituye en un instrumento fundamental para paliar el déficit habitacional existente en el Perú como lo señala Barco et al. (2009).

La morosidad, según la Superintendencia de Banca y Seguros y AFP (2003) está dividida por los días de incumplimiento, así como es caracterizada por las entidades bancarias.

- Categoría Normal: Los deudores que tienen un atraso de hasta ocho (8) días calendario.

- Categoría con Problemas Potenciales: Los deudores que tienen un atraso de nueve (9) a treinta (30) días calendario.

- Categoría Deficiente: Los deudores que tienen atraso de treinta y uno (31) a sesenta (60) días calendario. 
- Categoría Dudosa: Los deudores que tienen atraso de sesenta y uno (61) a ciento veinte (120) días calendario.

- Categoría Pérdida: los deudores que tienen atraso de más de ciento veinte (120) días calendario.

- Se considera un crédito vencido cuando tiene más de quince (15) días de atraso para los créditos corporativos, a grandes y a medianas empresas; más de treinta (30) días para los créditos a pequeñas y microempresas, y si mencionamos el caso de los créditos hipotecarios y de consumo, se considera vencida la cuota con más de treinta (30) días de atraso y el saldo a partir de noventa (90) días de atraso.

La Intermediación bancaria, según Delgado (2012) se da por medio de la captación de los depósitos del público para ser colocados como créditos al público en general. Es acá donde se genera la posibilidad de un no pago, que se traduce en mora porque el captador de los fondos no puede pagar al colocador de los mismos. Es por ello, que una función del banco es que coincidan los acreedores con los prestatarios, los mismos que pueden ser personas, hogares, empresas financieras y no financieras, gobiernos locales y nacionales.

La Regulación bancaria, tiene como base el poder limitar la exposición de los bancos a los riesgos de crédito, mercado, liquidez y solvencia. Para ello, se puede mencionar que los bancos deben mantener un capital mayor para poder amortiguar perdida futura inesperada y así absorber el impacto negativo de algún suceso (Gobat, 2012).

La solidez de un banco es importante por eso se toman medidas para evitar la quiebra del mismo. Para ello, los bancos tienen respaldo del gobierno a través de préstamos de emergencia por parte del banco central, además estas instituciones por intermediar dinero del público están sometidas, reguladas y supervisadas constantemente, para generar la confianza del público y facilitar el dinamismo de la economía, por su generación de dinero.

Existen autores que indican que los factores explicativos del comportamiento de la morosidad, son los siguientes: alta sensibilidad con respecto al ciclo económico (Freixas et al., 1994), es así que ante un mayor nivel de crecimiento económico los agentes disponen de un mayor nivel de ingreso para consumo. Los mismos autores expresan que los tipos de interés son otro factor, debido a que mientras mejores sean las tasas de interés para los deudores con créditos denominados en cualquier moneda, generaran menores cuantías en las cuotas de los préstamos.

Altos niveles de endeudamiento, ello está relacionado a límites que definen los ingresos familiares de los deudores de manera que si sobrepasan esos límites, les será no viable a mediano, largo plazo honrar sus obligaciones a menos que sus ingresos aumenten.

Otros autores también sostienen que existen otros factores:
Crecimiento en el nivel de renta disponible, según Vallcorba \& Delgado (2007), ante un mayor nivel de ingreso para las familias estas podrán disponer de una proporción, que si bien en términos relativos será equivalente al pago de sus obligaciones, debido a sus mejores niveles de ingreso será mayor en términos absolutos, lo que les permitirá afrontar con mayor holgura sus deudas.

Depreciación de la moneda local, según Azabache (2009), si se presentan variaciones en la moneda local respecto a la extranjera puede llevar a cierto riesgo en el cambio de moneda, que ocasionaría que los prestamos crezcan incrementándose la dificultad en capacidad de pago, si los ingresos no crecen.

Falta de una adecuada inteligencia financiera, con el fin de segmentar a la población por grupos de distinto riesgo de manera adecuada, evitando así un problema de selección adversa (Aguilar et al., 2004).

\section{Determinantes de la Morosidad bancaria en una economía dolarizada. Caso de Uruguay}

Vallcorba \& Delgado (2007), estudiaron los determinantes de la morosidad bancaria crediticia en Uruguay, con el fin de evaluar si existe una relación de equilibrio a largo plazo entre la morosidad y un conjunto de variables macroeconómicas seleccionadas. Ellos manifestaron que existe una relación entre la morosidad y sus rezagos, el desempleo y los tipos de interés rezagados en " $n$ " períodos si es positiva esta relación a un mayor nivel en dichas variables, el nivel de morosidad actual tendera ser mayor, mientras que la relación entre la morosidad y el nivel de la actividad económica, el crédito y las garantías rezagadas en "n" períodos es negativa significa que a un mayor nivel en dicha variables, el nivel de morosidad actual tendera a ser menor; por lo que muestran relaciones de codependencia a largo plazo. Finalmente concluyen que existe una relación de equilibrio a largo plazo entre morosidad y la variación de los salarios y los tipos de interés denominados en $\mathrm{MN}$, así como en ME.

Algo similar afirmaron Delgado \& Saurina (2004) quienes sostienen que existe una relación entre las variables macroeconómicas y el riesgo de crédito de las entidades de depósito españolas.

\section{Racionamiento del crédito en el mercado con información imperfecta}

La simetría de la información existente en las transacciones entre agentes deficitarios y superavitarios, que se presentan en los mercados de crédito producen un resultado ineficiente ya que la cantidad de créditos otorgados es menor a la que debería otorgarse (a través del aumento en las tasas de interés), reduciendo así la función agregada de bienestar social. Stilitz \& Weiss (1981); Muñoz, (1999); Vega y Vega (2012), sostienen que mientras la tasa de interés aumenta 
(debido a la existencia de información imperfecta), hace que la cantidad de individuos que no pueden acceder a los créditos aumenta. Existiendo una relación positiva entre la tasa de interés y la cantidad de personas que no acceden al crédito debido a la existencia de información imperfecta.

\section{Análisis de la Morosidad en el sistema Bancario Peruano}

Aguilar et al. (2004) buscaron identificar las variables que afectan el nivel de morosidad del sistema bancario, evaluando el impacto tanto de las variables de carácter agregado o macroeconómico (PBI, riesgo país, inflación, entre otros) como de aquellas relacionadas con la gestión de cada entidad financiera (política de créditos, diversificación del riesgo, entre otras). Concluyendo que la calidad de la cartera de las colocaciones en el Perú se relaciona de forma negativa con el ciclo de la actividad económica, mientras que concluyen que una devaluación real puede afectar seriamente la morosidad debido al descalce de activos y pasivos que tendrían los agentes económicos.

\section{Un estudio panel para el caso de las cajas municipales de ahorro y crédito del Perú}

Cermeño et al. (2011) investigaron los determinantes de la morosidad de la cartera en el sistema de cajas municipales del Perú, mediante el uso de un modelo de panel data con efectos fijos. Muestran la relación de la calidad de la cartera con la tasa de interés de los créditos, la liquidez en moneda nacional y el ratio de depósitos de créditos debería ser positiva debido a que un mayor nivel en dichas variables, el nivel de morosidad actual tenderá a ser mayor dado que los problemas de simetría inducirán a asignaciones ineficientes de recursos traduciéndose en un empeoramiento de la calidad de la cartera, mientras que la relación entre la calidad de la cartera y la variación del PBI se espera que sea negativa debido a que a mayor nivel de la actividad económica, la calidad de la cartera deberá mejorar.

\section{Efectos no lineales de las variaciones del tipo de cambio sobre el riesgo cambiario-crediticio. Evidencia empírica para el Perú}

Azabache (2009) evaluó el impacto de las variaciones del tipo de cambio sobre el riesgo cambiario-crediticio de los bancos a través de un modelo umbral. A través de él, se buscó estimar el nivel umbral de depreciación mediante el cual, a través de la variación del tipo de cambio, la morosidad del portafolio de créditos denominados en dólares se incrementara fuertemente.

\section{Determinantes de la morosidad de la cartera en el sistema financiero de Colombia}

Busca evaluar los resultados para determinar si en el caso colombianos hay relación de equilibrio de largo plazo entre la cartera de créditos y la cartera vencida de los mismos, si existe causalidad entre la cartera y su calidad futura y si la cartera vencida es sensible a choques de la cartera de créditos. Es así que el modelo considera factores macro como el desempeño de la economía, medidos con variables proxy como el índice de producción industrial y la demanda nacional de energía, las variables monetarias medida con la tasa de interés real; y las microeconómicas que incluyen el tamaño relativo del banco medido como el índice de participación de su cartera en el sistema; la eficiencia medida como el nivel de los gastos administrativos, que comprende los gastos de personal, gastos generales, depreciaciones y amortizaciones; y la cobertura de garantías, medido como el monto de los créditos sin garantías admisibles.

\section{Propuesta de modelo}

A partir de los modelos antes señalados se elaboró un modelo que busca explicar la morosidad a través de variables macroeconómicas, tales como la variación del PBI real, la tasa activa en moneda nacional, la tasa activa en moneda extranjera la variación del tipo de cambio real.

Mor $_{t}=\alpha_{t}+\beta_{1 t}$ VPBI $_{t}+\beta_{2 t}$ TAMN $_{t}+\beta_{3 t}$ TAMEX $_{t}+\beta_{4 t} \mathrm{VTCr}_{t}+\varepsilon_{t}$

Definición de cada una de las variables:

- Morosidad Crediticia (Mor), es el cociente de la cantidad de créditos atrasados o judicializados sobre el total de la cartera de colocaciones, es un reflejo de la calidad de cartera que posee una institución financiera en términos de exposición al riesgo de crédito.

- Nivel de Actividad Económica (VPBI), se refiere a la variación interanual del valor total de la producción corriente de bienes y servicios finales dentro del territorio nacional. Dicha relación causal esperada sería negativa o inversa con respecto a la morosidad crediticia hipotecaria, debido a que ante un mayor nivel de actividad económica los agentes económicos cuentan con un mayor nivel de ingresos disponible

- Depreciación del Tipo de Cambio real (VTCr), se refiere a la pérdida de valor de la moneda nacional respecto a una divisa fuerte como es el caso del dólar americano, la relación causal esperada es positiva, dado que una abrupta depreciación del tipo de cambio afectaría la capacidad de pago de los agentes con deuda denominada en moneda extranjera (efecto descalce entre los activos en moneda nacional, y los pasivo en moneda extranjera).

- Tasa de Interés Activa Promedio en Moneda Nacional (TAMN), se refiere al interés porcentual que las instituciones financieras cobran a los deudores por sus deudas denominadas en moneda nacional. La relación esperada basada en el marco teórico conceptual se espera que sea positiva.

- Tasa de Interés Activa Promedio en Moneda Extranjera (TAMEX), se refiere al interés porcentual que las instituciones en moneda extranjera. La relación 
esperada basada en el marco teórico conceptual debería ser positiva.

Debido a que el análisis de estudio se realizó a partir del año 2001, se tomaron en cuenta las entidades financieras que operan desde 2001, ellas son: Banco Citibank, Banco de Comercio, Banco de Crédito del Perú, Banco Financiero, Banco Interamericano de Finanzas, Banco Scotiabank Perú, Banco Interbank, Banco Mi Banco, Banco Falabella del Perú y Banco Continental.

\section{Resultados y discusión}

\section{Contrastaciones Empíricas}

\section{Pruebas y contraste de las series}

Se realizó la prueba raíz unitaria para la variable endógena: Morosidad Hipotecaria

Tabla 1. Dickey-Fuller

Dfuller lmorhip, Dickey-Fuller test for unit root, Number of obs $=179$

\begin{tabular}{ccccc}
\hline & & \multicolumn{3}{c}{ Interpolated Dickey-Fuller } \\
\hline & Test & $1 \%$ Critical & $5 \%$ Critical & $10 \%$ Critical \\
& Statistic & Value & Value & Value \\
\hline $\mathrm{Z}(\mathrm{t})$ & $-3,348$ & $-3,484$ & $-2,885$ & $-2,575$ \\
\hline
\end{tabular}

$\mathrm{H}_{0}$ : La serie presenta raíz unitaria

$\mathrm{H}_{0}$ : la serie está generada por un proceso estacionario

Como p-value $<0,05$, se rechaza H0, lo que implica que la serie Imorhip no tiene raíz unitaria y esta generada por un proceso estacionario.

Tabla 2. Phillips_Perron

Pperrom Itamn, Phillips-Perron test for unit root, Number of obs $=179$, Newey-Est lags $=4$

\begin{tabular}{ccccc}
\hline & \multicolumn{4}{c}{ Interpolated Dickey-Fuller } \\
\cline { 2 - 5 } & Test & $\begin{array}{c}1 \% \text { Critical } \\
\text { Vtatistic }\end{array}$ & $\begin{array}{c}5 \% \text { Critical } \\
\text { Value }\end{array}$ & $\begin{array}{c}\text { Value } \\
\text { Valical }\end{array}$ \\
\hline $\mathrm{Z}(\mathrm{rho})$ & $-1,141$ & $-20,063$ & $-13,858$ & $-11,105$ \\
$\mathrm{Z}(\mathrm{t})$ & $-0,618$ & -3.484 & $-2,885$ & $-2,575$
\end{tabular}

MacKinnon approximate $\mathrm{p}$-value for $\mathrm{Z}(\mathrm{t})=0,8668$

$\mathrm{H}_{0}$ : La serie presenta raíz unitaria

$\mathrm{H}^{0}$ : la serie está generada por un proceso estacionario

Como p-value $<0,05$, se falla en rechazar $\mathrm{H} 0$, lo que implica que la serie

Itamn tiene raíz unitaria y no esta generada por un proceso estacionario.

Tabla 3. Regresión (MCO) del modelo con series transformadas (Modelo Final) reg.lmorhip ldtc ldtamex VarPBIreal

\begin{tabular}{ccccccc}
\hline Source & SS & df & MS & Number of obs $=180$ \\
\hline Model & 78.8626258 & 4 & 19.7156564 & $\mathrm{~F}(4,175)=111.61$ \\
Residual & 30.9147007 & 175 & 0.176655433 & Prob $>\mathrm{F}=0.0000$ \\
Total & 109.777327 & 179 & 0.176688433 & \multicolumn{4}{c}{ R-squared $=0.7184$} \\
\hline & & \multicolumn{5}{c}{ Adj R-squared $=0.7120$} \\
& & & & \multicolumn{3}{c}{ Root MSE $=0.4203$} \\
\hline lmorhip & Coef. & Std. Err. & $\mathrm{t}$ & $\mathrm{P}>|\mathrm{t}|$ & [95\% Conf. interval] \\
1dtc & 6.123435 & 0.4514434 & 13.56 & 0.0000 & 5.232461 & 7.014409 \\
ldtam & -0.7714132 & 0.3599675 & -2.14 & 0.033 & -1.481849 & -0.0609768 \\
ldtamex & 0.7147005 & 0.3735725 & 1.91 & 0.057 & -0.0225869 & 1.451988 \\
VarPBIreal & -3.647936 & 1.038991 & -3.51 & 0.001 & -5.698501 & -1.597371 \\
-cons & -10.31202 & 1.084445 & -9.51 & 0.0000 & -12.45229 & -8.171744 \\
\hline
\end{tabular}

\section{Análisis Global del Modelo}

\section{$R$ cuadrado y $R$ ajustado}

El coeficiente de determinación (R-Squared) del modelo es 0.7120 , está dentro del rango 0 y 1 considerablemente al límite superior del mismo. Por lo tanto explica, significativamente a la variable morosidad crédito hipotecario.

- Prob F- Prueba F:

Con respect a la prueba de significancia conjunta, la especificación del modelo compuesta por el conjunto de variables, se muestra correcta, debido a que la prob. $>\mathrm{F}$ es de 0.0000 , lo que el conjunto de variables, explican significativamente a la morosidad crediticia hipotecaria.

\section{Análisis Individual de los coeficientes de regresión}

Los signos de las variable son consistentes con lo esperado por el modelo teórico propio excepto la TAMN.

\section{- $\quad$ VarPBIreal $=\mathbf{- 3 . 6 4 7 9 3 6}$}

Muestra la relación inversa con respecto a la morosidad, y que mediante una variación de 3.64 puntos porcentuales en el nivel de actividad económica, la morosidad variará en un punto porcentual. Con un p_value de $0.001<0.05$, la variable es estadísticamente significativa, mientras que es signo que muestra la relación entre la variable explicativa y la morosidad hipotecaria 8 variable explicada), es inversa y conssistente con la lógica económica del modelo teórico propio. Las pruebas de estacionalidad y los ajustes de las series 8 (de ser necesarias), se presentan a continuación:

Tabla 4. Dickey-Fuller

Dfuller VarPBIreal, Dicey-Fuller test for unit root, Number of obs $=179$

\begin{tabular}{lcccc}
\hline & & \multicolumn{3}{c}{ Interpolated Dickey-Fuller } \\
\cline { 2 - 5 } & Test Statistic & $\begin{array}{c}1 \% \text { Critical } \\
\text { Value }\end{array}$ & $\begin{array}{c}5 \% \text { Critical } \\
\text { Value }\end{array}$ & $\begin{array}{c}10 \% \text { Critical } \\
\text { Value }\end{array}$ \\
\hline $\mathrm{Z}(\mathrm{t})$ & -5.063 & -3.484 & -2.885 & -2.575 \\
\hline
\end{tabular}

MacKinnon approximate $\mathrm{p}$-value for $\mathrm{Z}(\mathrm{t})=0.0000$

$\mathrm{H}_{0}$ : La serie presenta raíz unitaria

$\mathrm{H}_{\mathrm{a}}$ : la serie está generada por un proceso estacionario Como p-value $<0.05$, se falla en rechazar $\mathrm{H} 0$, lo que implica que la serie VarPBIreal no tiene raíz unitaria y esta generada por un proceso estacionario.

\section{. $d \log \mathrm{TC}=6.123435$}

Muestra la relación directa con respecto a la morosidad, y que mediante una variación de $6.12 \%$ en el tipo de cambio (depreciación), la morosidad hipotecaria aumentará en 1 punto porcentual. Con un p_value de $0.033<0.05$, la variable es estadísticamente significativa, mientras que el signo que muestra la relación entre la variable explicativa y la morosidad hipotecaria (variable explicativa), es directa y consistente con la lógica económica del modelo teórico propio. 
Tabla 5. Phillips_Perron

Pperrom VarPBIreal, Phillips-Perron test for unit root, Number of obs $=179$, Newey-Est lags $=4$

\begin{tabular}{ccccc}
\hline & \multicolumn{4}{c}{ Interpolated Dickey-Fuller } \\
\cline { 2 - 5 } & Test & $1 \%$ Critical & $5 \%$ Critical & $10 \%$ Critical \\
& Statistic & Value & Value & Value \\
\hline $\mathrm{Z}($ rho) & -36.340 & -20.063 & -13.858 & -11.105 \\
$\mathrm{Z}(\mathrm{t})$ & -4.707 & -3.484 & -2.885 & -2.575 \\
\hline
\end{tabular}

MacKinnon approximate $\mathrm{p}$-value for $\mathrm{Z}(\mathrm{t})=0.0001$

$\mathrm{H}_{0}$ : La serie presenta raíz unitaria

$\mathrm{H}^{0}$ : la serie está generada por un proceso estacionario

Como p-value $<0.05$, se rechaza H0, lo que implica que la serie VarPBIreal no tiene raíz unitaria y esta generada por un proceso estacionario.

\section{$. d \operatorname{dog} T A M N=-\mathbf{0 . 7 7 1 4 1 3 2}$}

Muestra la relación inversa con respecto a la morosidad, y que mediante una variación de 77 puntos básicos en la Tasa Activa en Moneda Nacional, la morosidad variará en un punto porcentual. Con un $\mathrm{p}$ _value de $0.001<0.05$, la variables es estadísticamente significativa, mientras que es signo que muestra la relación entre la variable explicativa y la morosidad hipotecaria (variable explicada), es inversa; sin embargo, no se condice con la lógica esperada según el modelo teórico propio, ya que la relación es inversa lo que implicaría que una aumento en la tasa de interés activa en moneda nacional, disminuirá la morosidad crediticia hipotecaria, ello se podría explicar debido a que en el Perú las tasas de interés, en su mayoría, son fijas a diferencia de países con mayor grado de profundización financiera en los cuales las tasas suelen ser variables, por ejemplo: Libor + spread, lo que implica que ante un aumento de los tipos de interés el valor de las cuotas pactadas, aumenta ello dificulta la posibilidad de pago de los deudores hipotecrios, como lo ocurrido durante la crisis subprime del año 2007 (Maubré, 2008). Explicado lo anterior, se entiende que ante un aumento de las tasas de interés se generara un efecto de selección positiva, a la cual solo accederán clientes con mayor capacidad de pago (mayores niveles de ingresos), lo que explicaría la reducción de la morosidad ante la subida de las tasas de interés. Las pruebas de estacionalidad y los ajustes de las series, se presentan a continuación:

Tabla 6. Dickey-Fuller

dfuller ltamnn, Dickey-Fuller test for unit root, Number of obs $=179$

\begin{tabular}{lcccc}
\hline & \multicolumn{4}{c}{ Interpolated Dickey-Fuller } \\
\cline { 2 - 5 } & \multirow{2}{*}{ Test Statistic } & $\begin{array}{c}1 \% \text { Critical } \\
\text { Value }\end{array}$ & $\begin{array}{c}5 \% \text { Critical } \\
\text { Value }\end{array}$ & $\begin{array}{c}10 \% \text { Critical } \\
\text { Value }\end{array}$ \\
\cline { 2 - 5 } & -3.12 & -3.484 & -2.885 & -2.575 \\
\hline
\end{tabular}

MacKinnon approximate $\mathrm{p}$-value for $\mathrm{Z}(\mathrm{t})=0.9238$

$\mathrm{H}_{\mathrm{o}}$ : La serie presenta raíz unitaria

$\mathrm{H}_{\mathrm{a}}$ : la serie está generada por un proceso estacionario

Como p-value $<0.05$, se falla en rechazar H0, lo que implica que la serie Itamn tiene raíz unitaria y no esta generada por un proceso estacionario.

\section{Transformación en primera diferencia de ltamn a Idtamn}

Debido a que la serie ltamn muestra raíz unitaria se procedioa realizar un ajuste en primer diferencia de la misma.
Tabla 7. Dickey-Fuller

dfuller ltamn, Dickey-Fuller test for unit root, Number of obs $=178$

\begin{tabular}{lcccc}
\hline & \multicolumn{3}{c}{ Interpolated Dickey-Fuller } \\
\cline { 2 - 5 } & \multirow{2}{*}{ Test Statistic } & $\begin{array}{c}1 \% \text { Critical } \\
\text { Value }\end{array}$ & $\begin{array}{c}5 \% \text { Critical } \\
\text { Value }\end{array}$ & $\begin{array}{c}10 \% \text { Critical } \\
\text { Value }\end{array}$ \\
\hline$Z(\mathrm{t})$ & -10.348 & -3.484 & -2.885 & -2.575 \\
\hline
\end{tabular}

MacKinnon approximate $\mathrm{p}$-value for $\mathrm{Z}(\mathrm{t})=0.0000$

$\mathrm{H}_{0}$ : La serie presenta raíz unitaria

$\mathrm{H}_{\mathrm{a}}$ : la serie está generada por un proceso estacionario

$\mathrm{C}_{\mathrm{o}}^{\mathrm{a}}$ mo p-value $<0.05$, se rechaza $\mathrm{H}_{0}$, lo que implica que la serie transformada Idtamn no tiene raíz unitaria y esta generada por un proceso estacionario.

Tabla 8. Phillips_Perron

Pperrom ldtamn, Phillips-Perron test for unit root, Number of obs $=179$, Newey-Est lags $=4$

\begin{tabular}{ccccc}
\hline & \multicolumn{4}{c}{ Interpolated Dickey-Fuller } \\
\cline { 2 - 5 } & Test & 1\% Critical & $5 \%$ Critical & $10 \%$ Critical \\
& Statistic & Value & Value & Value \\
\hline$Z($ rho $)$ & -43.911 & -20.063 & -13.858 & -11.105 \\
$Z(\mathrm{t})$ & -10.498 & -3.484 & -2.885 & -2.575 \\
\hline
\end{tabular}

MacKinnon approximate $\mathrm{p}$-value for $\mathrm{Z}(\mathrm{t})=0.0000$

$\mathrm{H}_{0}$ : La serie presenta raíz unitaria

$\mathrm{H}_{\mathrm{a}}^{0}$ : la serie está generada por un proceso estacionario

Como p-value $<0.05$, se rechaza $\mathrm{H}_{0}$, lo que implica que la serie transformada ldtamn no tiene raíz unitaria y esta generada por un proceso estacionario.

Tabla 9. Phillips_Perron

Pperrom ltamn, Phillips-Perron test for unit root, Number of obs $=179$, Newey-Est lags $=4$

\begin{tabular}{ccccc}
\hline & \multicolumn{4}{c}{ Interpolated Dickey-Fuller } \\
\cline { 2 - 5 } & Test & 1\% Critical & 5\% Critical Value & 10\%Critical Value \\
\hline$Z($ Vhotistic & -1.141 & -20.063 & -13.858 & -11.105 \\
$Z(\mathrm{t})$ & -0.618 & -3.484 & -2.885 & -2.575 \\
\hline
\end{tabular}

MacKinnon approximate $\mathrm{p}$-value for $\mathrm{Z}(\mathrm{t})=0.8668$

$\mathrm{H}_{0}$ : La serie presenta raíz unitaria

$\mathrm{H}^{0}$ : la serie está generada por un proceso estacionario

Como p-value $<0.05$, se rechaza $\mathrm{H}_{0}$, lo que implica que la serie ltamn tiene raíz unitaria y no esta generada por un proceso estacionario.

\section{. dlogTAМЕX $==.7147005$}

Muestra la relación directa con respecto a la morosidad, y que mediante una variación de 71 puntos básicos en la Tasa Activa denominada en Moneda Extranjera, la morosidad hipotecaria aumentara en 1 punto porcentual. Con un p_value de $0.056>0.1$, la variable es estadísticamente significativa, mientras que es signo que muestra la relación entre la variable explicativa y la morosidad hipotecaria (variable explicada), es directa y consistente con la lógica económica del modelo teórico propio.

Tabla 10. Dickey-Fuller

dfuller ltamex, Dickey-Fuller test for unit root, Number of obs $=179$

\begin{tabular}{ccccc}
\hline & \multicolumn{3}{c}{ Interpolated Dickey-Fuller } \\
\hline \multirow{2}{*}{$\mathrm{Z}(\mathrm{t})$} & Test Statistic & $\begin{array}{c}1 \% \text { Critical } \\
\text { Value }\end{array}$ & $\begin{array}{c}5 \% \text { Critical } \\
\text { Value }\end{array}$ & $10 \%$ Critical Value \\
\cline { 2 - 5 } & -2.198 & -3.484 & -2.885 & -2.575 \\
\hline
\end{tabular}

MacKinnon approximate $\mathrm{p}$-value for $\mathrm{Z}(\mathrm{t})=0.2070$

$\mathrm{H}_{0}$ : La serie presenta raíz unitaria

$\mathrm{H}_{\mathrm{a}}$ : la serie está generada por un proceso estacionario

Como p-value $<0.05$, se falla en rechazar $\mathrm{H}_{0}$, lo que implica que la serie transformada Itamex tiene raíz unitaria y no esta generada por un proceso estacionario. 
Tabla 11. Phillips_Perron

Pperrom ltamex, Phillips-Perron test for unit root Number of obs $=179$, Newey-Wes lags $=4$

\begin{tabular}{ccccc}
\hline & \multicolumn{4}{c}{ Interpolated Dickey-Fuller } \\
\cline { 2 - 5 } & Test & $1 \%$ Critical & $5 \%$ Critical & $10 \%$ Critical \\
& Statistic & Value & Value & Value \\
\hline$Z($ rho $)$ & -3.837 & -20.063 & -13.858 & -11.105 \\
$Z(t)$ & -1.992 & -3.484 & -2.885 & -2.575 \\
\hline
\end{tabular}

MacKinnon approximate $\mathrm{p}$-value for $\mathrm{Z}(\mathrm{t})=0.2901$

$\mathrm{H}_{0}$ : La serie presenta raíz unitaria

$\mathrm{H}_{2}$ : la serie está generada por un proceso estacionario

Como p-value $<0.05$, se falla en rechazar $\mathrm{H}_{0}$, lo que implica que la serie transformada ltamex tiene raíz unitaria y no esta generada por un proceso estacionario.

\section{Transformación en primera diferencia de Itamex por Idtamex}

Debido a que la serie ltamex muestra raíz unitaria se procedió a realizar un ajuste en primera diferencia de la misma.

Pruebas de Raíz Unitaria para Var, Tansformada Idtamex

Tabla 12. Dickey-Fuller

dfuller ldtamex, Dickey-Fuller test for unit root, Number of obs $=178$

\begin{tabular}{ccccc}
\hline & \multicolumn{4}{c}{ Interpolated Dickey-Fuller } \\
\cline { 2 - 5 } & Test & $\begin{array}{c}\text { 1\% Critical } \\
\text { Statistic }\end{array}$ & $\begin{array}{c}5 \% \text { Critical } \\
\text { Value }\end{array}$ & $\begin{array}{c}10 \% \text { Critical } \\
\text { Value }\end{array}$ \\
\hline $\mathrm{Z}(\mathrm{t})$ & -7.042 & -3.484 & -2.885 & -2.575 \\
\hline
\end{tabular}

MacKinnon approximate $\mathrm{p}$-value for $\mathrm{Z}(\mathrm{t})=0.0000$

$\mathrm{H}_{0}$ : La serie presenta raíz unitaria

$\mathrm{H}^{0}$ : la serie está generada por un proceso estacionario

Como p-value $<0.05$, se rechaza $\mathrm{H}_{0}$, lo que implica que la serie transformada Idtamex no tiene raíz unitaria y no esta generada por un proceso estacionario.

Table 13. Phillips_Perron

.pperrom ldtamex

Phillips-Perron test for unit root, Number of obs $=178$, Newey-Wes lags $=4$

\begin{tabular}{ccccc}
\hline & \multicolumn{4}{c}{ Interpolated Dickey-Fuller } \\
\cline { 2 - 5 } & $\begin{array}{c}\text { Test } \\
\text { Statistic }\end{array}$ & $\begin{array}{c}\text { 1\% Critical } \\
\text { Value }\end{array}$ & 5\% Critical Value & $\begin{array}{c}\text { 10\%Critical } \\
\text { Value }\end{array}$ \\
\hline $\mathrm{Z}(\mathrm{rho})$ & -76.261 & -20.063 & -13.858 & -11.105 \\
$\mathrm{Z}(\mathrm{t})$ & -6.968 & -3.484 & -2.885 & -2.575 \\
\hline
\end{tabular}

MacKinnon approximate $\mathrm{p}$-value for $\mathrm{Z}(\mathrm{t})=0.0000$

$\mathrm{H}_{0}$ : La serie presenta raíz unitaria

$\mathrm{H}^{0}$ : la serie está generada por un proceso estacionario

Como p-value $<0.05$, se rechaza $\mathrm{H}_{0}$, lo que implica que la serie transformada ldtamex no tiene raíz unitaria y no esta generada por un proceso estacionario.

\section{Interpretación respecto al modelo teórico propio}

El modelo econométrico basado en el propuesto, muestra resultados consistentes con lo esperado, en términos de significancia económica y estadística, la determinación y significancia conjunta del modelo muestra que este es adecuado para estimar niveles de morosidad crediticia hipotecaria, en función de los determinantes macroeconómicos tales como: el nivel de actividad económica, depreciación del tipo de cambio, tasas de interés activas en moneda nacional y extranjera. Mostrándose que los más significativos para la determinación de la morosidad crediticia hipotecaria, son el nivel de actividad económica y la variación del tipo de cambio. Lo que implica que si existe un deterioro del ciclo económico y/o de una abrupta depreciación del tipo de cambio, inevitablemente habrá un deterioro de la calidad de la cartera del portafolio de créditos hipotecarios, denominado en moneda nacional así como extranjera.

\section{Conclusiones y recomendaciones}

Las variables macroeconómicas tales como crecimiento económico, tasas de interés y tipo de cambio influyen en el comportamiento de la calidad de la cartera de créditos hipotecarios. En el trabajo se muestra que la morosidad crediticia hipotecaria de la banca múltiple en el Perú medido por el ratio de morosidad crediticia, ha disminuido debido a la mejora de los factores económicos antes señalados. La calidad de la cartera hipotecaria se relaciona positivamente con el ciclo económico. Es así, que en ciclos de auge y tienden a mejorar la calidad de los créditos, mientras ocurre lo contrario en las fases recesivas.

Existe un $21 \%$ de las colocaciones de la cartera hipotecaria que aún están en moneda extranjera, lo cual hace que el comportamiento del tipo de cambio sea un factor macroeconómico importante para explicar la calidad de la cartera. Una devaluación abrupta puede afectar seriamente la morosidad debido al descalce de activos y pasivos que presentarían los agentes económicos que tiene ingresos en soles, pero obligaciones en dólares, por otro lado, incrementos en el tipo de cambio real pueden estar asociados a que los precios domésticos suban más rápido que los de nuestros socios comerciales, lo que lleva al deterioro de la capacidad adquisitiva local.

Se recomienda hacer el análisis de la variación del tipo de cambio en periodos más cortos para determinar en que coyuntura y cuáles son las variables que determinan cuando una abrupta depreciación del tipo de cambio afecta significativamente la calidad del portafolio den moneda extranjera.

\section{Literatura citada}

Aguilar, G.; Camargo, G. \& Morales, R. 2004. Análisis de la morosidad en el sistema bancario peruano. Informe Final de Investigación. Instituto de Estudios PeruanosIEP, Octubre, 2004.

Azabache, P. 2009. Efectos no lineales de las variaciones del tipo de cambio sobre el riesgo cambiario-crediticio. Evidencia empírica para el Perú. Revista Estudios Económicos 18. BCRP.

Barco, D.; Ibérico, J. y Medina, A. 2009. Situación del Crédito Hipotecario en el Perú en Financiamiento de la Vivienda, Revista Moneda 140. BCRP.

Cermeño, R.; León, J. y Mantilla, G. 2011. Determinantes de la morosidad: Un estudio panel para el caso de las cajas municipales de ahorro y crédito del Perú, 2003- 
2010. Documentos de Trabajo, Centro de Investigación y Docencia Económica - CIDE, N 513, Nov., Mexico.

Chavarín, R. 2015. Morosidad en el pago de créditos y rentabilidad de la banca comercial en México. Revista Mexicana Economía y Finanzas 10 (1): 73-85.

Damodar, G. \& Dawn, P. 2010. Essentials of Econometrics. 4Th Edition Mc Graw - Hill. Irwin.

Delgado, M. 2012. Determinantes de la morosidad del sistema bancario 2001-2008.

Delgado, J. y Saurina, J. 2004. Riesgo de crédito y dotaciones a insolvencias. Un análisis con variables macroeconómicas. Recuperado de: http://w.w.w.repositoriocdpd.net:8080/bitstream/ handle/123456789/986/Art_MaloMA_DibleDiscrimin acionSalarial_2007.pdf)sequence $=1$.

Freixas, X.; Hevia, J. \& Inurrieta, A. 1994. Determinantes macroeconómicos de la morosidad bancaria: un modelo empírico para el caso español. Moneda y Crédito, Editorial Fundación Santander Central Hispano $\mathrm{N}^{\mathrm{o}}$ 204.

Gobat, J. 2012. ¿Qué es un banco?. Recuperado de: http:// www.imf.org/external/pubs/ft/fandd/spa/2012/03/pdf/ basics.pdf.

Guillen, J. 2001. Morosidad crediticia y tamaño. Un análisis de la crisis bancaria peruana. Revista Documentos de Trabajo $\mathrm{N}^{\mathrm{o}} 7$, BCRP.

Maubré, J. 2008. La crisis de los créditos subprime. Coqueteando con el riesgo. Revista Ejecutivos de Finanzas, Abril/ mayo: 28-40.

Muñoz, J. 1999. Calidad de cartera del sistema bancario y el ciclo económico: Una aproximación econométrica para el caso peruano. Revista Estudios Económicos $\mathrm{N}^{\mathrm{o}}$ 4 BCRP.

Stilitz J. \& weiss A. 1981. Credit rationing in Markets with Imperfect Information,. The American Economic Review 71 (3): 393-410.

Superintendencia de Banca, Seguros y Administradoras de Fondos de Pensiones (SBS) 2016. Memoria Institucional. Recuperado de: http://www.sbs.gob. pe/Portals/0/jer/PUB_MEMORIAS/20170712_SBSMemoria-2016.pdf

Superintencia de Banca y Seguros, y AFP (2003). Reglamento para la evaluación y clasificación del deudor y la exigencia de provisiones. Resolución S.B.S. No 808-2003. 28 de mayo del 2003.

Tanzi, V. 2010. La crisis financiera y económica de 20082009: efectos fiscales y monetarios. Madrid.

Vallcorba, M. \& Delgado, J. 2007. Determinantes de la Morosidad Bancaria en una economía dolarizada. El caso uruguayo. Documento de Trabajo No 0722 . Banco de España. Eurosistema, Madrid 2007.

Vega, H. \& Vega, M. 2012. Intermediación Financiera y Macroeconomía. Fricciones Financieras, Revista Moneda 50: 20-23. 